\title{
Gauged Nonlinear Sigma Model and Boundary Diffeomorphism Algebra
}

\author{
Phillial Oh* \\ Department of Physics and Basic Science Research Institute \\ Sung Kyun Kwan University, Suwon 440-746, Korea
}

\begin{abstract}
We consider Chern-Simons gauged nonlinear sigma model with boundary which has a manifest bulk diffeomorphism invariance. We find that the Gauss's law can be solved explicitly when the nonlinear sigma model is defined on the Hermitian symmetric space, and the original bulk theory completely reduces to a boundary nonlinear sigma model with the target space of Hermitian symmetric space. We also study the symplectic structure, compute the diffeomorphism algebra on the boundary, and find an (enlarged) Virasoro algebra with classical central term.
\end{abstract}

*Email address: ploh@dirac.skku.ac.kr 


\section{INTRODUCTION}

The Chern-Simons (CS) gravity theory [1] with boundary [2] has attracted a lot of recent attention in relation with black hole physics [3]. In particular, it has been observed [4] that the Virasoro algebra which lives on the boundary and carries classical central charge [5 6] may provide important clues in understanding the microscopic origin of black hole entropy.

It is well known that in the Chern-Simons theory [8], the Gauss constraint can be solved explicitly by the pure gauge condition, and the bulk theory completely reduces to a boundary chiral WZW model on $G$ [9]. If one imposes some extra condition on the group element, reduction from the target space of $G$ to that of $G / H$ occurs [10. For example, if one imposes an extra condition like $g^{2}=1, C P(N)$ nonlinear sigma model (NLSM) can be obtained. However, such process is ad hoc and the reduced theory on $C P(N)$ has some properties which cannot be obtained from the original WZW theory by simply substituting $g^{2}=1$ (The enlarged Virasoro algebra of eq. (29) is one such example.). The purpose of this Letter is to invent some scheme where the reduction to $G / H$ occurs directly as a consequence of Gauss' law constraint. Using the coadjoint orbit method for NLSM [11], we introduce a model in which the Chern-Simons gauge field and NLSM on $G / H$ have topological and gauge invariant interaction. Gauss's law can be solved explicitly when the NLSM is defined on the Hermitian symmetric spaces, and the original bulk theory completely reduces to a boundary NLSM with the target space of Hermitian symmetric space. We also study the symplectic structure of the boundary theory and compute the diffeomorphism algebra by the standard Noether procedure. We find that the diffeomorphism algebra becomes the Virasoro algebra with classical central term if a suitable boundary condition is satisfied. We also discover an enlarged Virasoro algebra with elements of symmetric tensor product.

\section{SYMPLECTIC REDUCTION AND BOUNDARY NLSM}

To describe the coupling of CS theory with NLSM, we introduce a coadjoint orbit variable $Q=g K g^{-1}$ on $G / H$, where $g \in G$ and $K \in \mathcal{H}$ is the centralizer for the Lie algebra $\mathcal{H}$. Then, we consider the Chern-Simons theory coupled with NLSM in a diffeomorphism invariant manner on the disc $D$

$$
L=\Gamma \int_{D} d^{2} x \epsilon^{\mu \nu \rho}\left\langle\left[Q, D_{\mu} Q\right] F_{\nu \rho}\right\rangle+\frac{\kappa}{2 \pi} \int_{D} d^{2} x \epsilon^{\mu \nu \rho}\left\langle A_{\mu} \partial_{\nu} A_{\rho}+\frac{2}{3} A_{\mu} A_{\nu} A_{\rho}\right\rangle,
$$

where $\langle\cdots\rangle$ denotes trace and $D_{\mu}=\partial_{\mu}+A_{\mu}$. Since we are interested in a complete reduction to the boundary theory, we neglect the metric dependent part of the Lagrangian for the variable $Q$ and consider only the manifest diffeomorphism-invariant theory. The above Lagrangian also has the gauge invariance

$$
g \rightarrow h g, Q \rightarrow h Q h^{-1}, A \rightarrow h A h^{-1}+h d h^{-1}, \quad(h \in G)
$$

and the constant coefficient $\Gamma(\equiv \kappa \alpha / 2 \pi)$ is not quantized. It will be related with the solution of the Gauss's law constraint. The Lagrangian also has a local symmetry $g \rightarrow$ $g h(h \in H), Q \rightarrow Q, A \rightarrow A$, which makes the field $Q$ to take values in the homogeneous space $G / H$. Up to a boundary term, (1) can be put into the following canonical form 


$$
L=\frac{\kappa}{4 \pi} \int_{D} d^{2} x \epsilon^{i j}\left(\left(A_{i}^{a}+2 \alpha\left[Q, D_{i} Q\right]\right) \dot{A}_{j}^{a}+\alpha\left[Q, F_{i j}\right]^{a} \dot{Q}^{a}-A_{0}^{a} G_{i j}^{a}\right) .
$$

(Here, $\epsilon^{012} \equiv \epsilon^{12} \equiv 1, Q=Q^{a} t^{a}, A_{i}=A_{i}^{a} t^{a}, F_{i j}=F_{i j}^{a} t^{a}, F_{i j}^{a}=\partial_{i} A_{j}^{a}-\partial_{j} A_{i}^{a}+f^{a b c} A_{i}^{b} A_{j}^{c}$, and the group generators $t^{a}$ satisfy $\left[t^{a}, t^{b}\right]=f^{a b c} t^{c},\left\langle t^{a} t^{b}\right\rangle=-\frac{1}{2} \delta^{a b}$.) $G_{i j}^{a}$ is the Gauss' law constraint given by

$$
G_{i j}^{a}=F_{i j}^{a}+2 \alpha\left[D_{i} Q, D_{j} Q\right]^{a}-2 \alpha\left[Q,\left[Q, F_{i j}\right]\right]^{a} .
$$

We shall take (3) as our starting point. Variation with respect to $A_{0}^{a}$ gives the Gauss' law constraint $G_{i j}^{a}=0$. We adopt the Hamiltonian symplectic method [12] and solve the constraint from the beginning. It turns out that the above constraint can be solved explicitly when $Q$ corresponds to Hermitian symmetric spaces [13,11] with the following Ansatz;

$$
A_{i}^{a}=c\left[Q, \partial_{i} Q\right]^{a}
$$

where $c$ is a constant to be determined. Substituting into (价) and using the identities $\left[Q,\left[Q, \partial_{i} Q\right]\right]=-\partial_{i} Q,\left[Q,\left[\partial_{i} Q, \partial_{j} Q\right]\right]=0$ which are satisfied [11] on Hermitian symmetric spaces, we find that the constraint can be solved by choosing

$$
c=-1 \mp \sqrt{1 /(1+2 \alpha)} . \quad\left(\alpha>-\frac{1}{2}\right)
$$

When $\alpha=0, c$ can be either 0 or -2 . In the latter case, $A_{i}^{a}=-2\left[Q, \partial_{i} Q\right]^{a}$ satisfies the zero curvature condition which in $S U(2)$ case has been studied before in the context of vacuum structure of pure Yang-Mills theory [14] and in integrable models [11]. Substitution into the Lagrangian (3) gives

$$
L=\gamma \oint d \varphi<\partial_{\varphi} Q \dot{Q}>
$$

where $\varphi$ denotes the angular coordinate on the boundary $\partial D$ of disc $D$ and $\gamma=\kappa c(2 \pi)^{-1}(c+$ $2 \alpha(1+c))$. Hence, the bulk theory has completely reduced to boundary theory upon substitution of the solution of the Gauss' law constraint [15.

In order to compute the symplectic structure, we follow the approach of Ref. [16] to rewrite the Lagrangian in terms of the current defined by $J_{\varphi}^{a}=\gamma\left[Q, \partial_{\varphi} Q\right]^{a}$. Using the identities $\left[Q,\left[Q, \partial_{\varphi} Q\right]\right]=-\partial_{\varphi} Q$, we obtain

$$
L=\oint d \varphi<J_{\varphi}[Q, \dot{Q}]>.
$$

which suggests the following canonical 1-form

$$
\theta=\oint d \varphi<J_{\varphi}[Q, d Q]>
$$

Then, using $[Q,[d Q, d Q]]=0$, we obtain (introducing the $Q^{\alpha \beta}$ and $J^{\gamma \delta}$ as the matrix element of $Q$ and $J$ )

$$
\omega=d \theta \equiv \frac{1}{2} \oint d \varphi \oint d \varphi^{\prime} d J^{\alpha \beta}(\varphi) \Omega_{\beta \delta ; \gamma \alpha}\left(\varphi, \varphi^{\prime}\right) d Q_{\varphi}^{\gamma \delta}\left(\varphi^{\prime}\right)
$$


where

$$
\Omega_{\beta \delta ; \gamma \alpha}\left(\varphi, \varphi^{\prime}\right)=2\left(-\delta^{\beta \gamma} Q^{\delta \alpha}+\delta^{\alpha \delta} Q^{\beta \gamma}\right) \delta\left(\varphi-\varphi^{\prime}\right)
$$

Introducing the tensor notation $(A \otimes B)_{\alpha \beta ; \gamma \delta}=A_{\alpha \gamma} B_{\beta \delta}$, we have $\Omega=2(Q \otimes I-I \otimes Q)$. The notation is to be understood as including the Dirac delta-function. Also, in order to simplify the calculation, we transform the original $Q \in \mathcal{G}$ into $Q^{2}=-I / 4$ which always can be achieved by a suitable addition of constant [11]. Note that adding a constant to $Q$ does not change the action (7), the current $J_{\varphi}^{a}$, and the symplectic structure (11).

In general, the presymplectic form $\Omega$ of (10) and (11) is degenerate and we must calculate the Poisson bracket on the reduced phase space. In order to achieve this, we introduce the projection operator $P$ and the inverse $\Omega^{-1}$ which is defined on the reduced phase space, and satisfy the following relations 17

$$
\Omega \Omega^{-1}=I \otimes I-P, \quad P^{2}=P, \quad P \Omega=\Omega^{-1} P=0 .
$$

Using $\Omega=2(Q \otimes I-I \otimes Q)$, we find the following solution of the above equation

$$
\Omega^{-1}=\frac{1}{2}(I \otimes Q-Q \otimes I), \quad P=\frac{1}{2}(I \otimes I-4 Q \otimes Q) .
$$

The above analysis yields the following Poisson bracket

$$
\left\{J^{\alpha \beta}(\varphi), Q^{\gamma \delta}\left(\varphi^{\prime}\right)\right\}=\Omega_{\gamma \alpha ; \beta \delta}^{-1}\left(\varphi, \varphi^{\prime}\right)=\frac{1}{2}\left(\delta^{\beta \gamma} Q^{\alpha \delta}-\delta^{\alpha \delta} Q^{\gamma \beta}\right) \delta\left(\varphi-\varphi^{\prime}\right),
$$

or in generator form

$$
\left\{J_{\varphi}^{a}(\varphi), Q^{b}\left(\varphi^{\prime}\right)\right\}=f^{a b c} Q^{c}(\varphi) \delta\left(\varphi-\varphi^{\prime}\right) .
$$

Then, we compute the bracket $\left\{J_{\varphi}^{a}(\varphi), J_{\varphi}^{b}\left(\varphi^{\prime}\right)\right\}$. In order to properly antisymmetrize with respect to the interchange of $(a, \varphi) \leftrightarrow\left(b, \varphi^{\prime}\right)$, we calculate

$$
\begin{aligned}
\left\{J_{\varphi}^{a}(\varphi), J_{\varphi}^{b}\left(\varphi^{\prime}\right)\right\} & =\gamma \frac{1}{2}\left(\left\{J_{\varphi}^{a}(\varphi), f^{b c d} Q^{c}\left(\varphi^{\prime}\right) \partial_{\varphi^{\prime}} Q^{d}\left(\varphi^{\prime}\right)\right\}+\left\{f^{a c d} Q^{c}(\varphi) \partial_{\varphi} Q^{d}(\varphi), J_{\varphi}^{b}\left(\varphi^{\prime}\right)\right\}\right) \\
& =f^{a b c} J_{\varphi}^{c}(\varphi) \delta\left(\varphi-\varphi^{\prime}\right)+\frac{\gamma}{2}\left(J^{a b}(\varphi)+J^{a b}\left(\varphi^{\prime}\right)\right) \delta^{\prime}\left(\varphi-\varphi^{\prime}\right)
\end{aligned}
$$

where ${ }^{\prime}=\partial / \partial \varphi$ and we defined the symmetric tensor product

$$
J^{a b}(\varphi)=f^{a c e} f^{b d e} Q_{c}(\varphi) Q_{d}(\varphi) .
$$

Also, one can show, using the Jacobi identities for the structure constants,

$$
\left\{J_{\varphi}^{a}(\varphi), J^{b c}\left(\varphi^{\prime}\right)\right\}=\left(f^{a b d} J^{d c}(\varphi)+f^{a c d} J^{b d}(\varphi)\right) \delta\left(\varphi-\varphi^{\prime}\right) .
$$

$J^{a}$ and $J^{b c}$ form an enlarged current algebra. This type of current algebra (116) (17) (18) ) first appeared in the analysis of $O(N)$ nonlinear sigma model through the Dirac constraint analysis [18. Later, it was generalized to arbitrary Riemannian manifolds with unconstrained variables using the Killing symmetry [19]. 


\section{DIFFEOMORPHISM ALGEBRA}

We first compute diffeomorphism (Diff) algebra directly from the action (17). Under diffeomorphism $\delta_{f} Q^{a}=f^{\varphi} \partial_{\varphi} Q^{a}, \delta_{f} L=0$, and the Noether charge becomes

$$
C(f)=\frac{\partial L}{\partial \dot{Q}^{a}} \delta_{f} Q^{a}=\gamma^{-1} \oint_{\partial D} d \varphi f^{\varphi}<J_{\varphi} J_{\varphi}>,
$$

where one used $\gamma^{2}<\partial_{\varphi} Q \partial_{\varphi} Q>=<J_{\varphi} J_{\varphi}>$. It is more convenient to use the matrix expression of (16) from here on;

$$
\begin{aligned}
\left\{J^{\alpha \beta}(\varphi), J^{\gamma \delta}\left(\varphi^{\prime}\right)\right\} & =\frac{1}{2}\left(\delta^{\beta \gamma} J^{\alpha \delta}-\delta^{\alpha \delta} J^{\gamma \beta}\right)(\varphi) \delta\left(\varphi-\varphi^{\prime}\right) \\
& -\frac{\gamma}{2}\left(\frac{1}{2} \delta^{\beta \gamma} \delta^{\alpha \delta}+Q^{\alpha \delta}(\varphi) Q^{\gamma \beta}(\varphi)+Q^{\alpha \delta}\left(\varphi^{\prime}\right) Q^{\gamma \beta}\left(\varphi^{\prime}\right)\right) \delta^{\prime}\left(\varphi-\varphi^{\prime}\right),
\end{aligned}
$$

Then, a straight forward computation produces the Virasoro algebra without a central charge

$$
\{C(f), C(g)\}=C([f, g]) .
$$

where $[f, g]=f^{\prime} g-f g^{\prime}$.

The above derivation of the Virasoro started directly from the reduced action on the boundary. However, one can also first compute Diff charge from the bulk action (3) by the Noether procedure, and then reduce it to a boundary Diff charge using the solution (5). As in the pure Chern-Simons case, the ensuing algebra depends on the boundary condition [6.16. We show that with a suitable choice of boundary condition, Diff algebra becomes the Virasoro algebra with classical central term. Let us start with the Lagrangian (3) and consider the response of $L$ to a spatial and time-independent diffeomorphism:

$$
\begin{aligned}
\delta_{f} x^{\mu} & =-\delta^{\mu}{ }_{i} f^{i}, \delta_{f} Q^{a}=f^{j} \partial_{j} Q^{a}, \\
\delta_{f} A_{i}^{a} & =f^{j} \partial_{j} A_{i}^{a}+\left(\partial_{i} f^{j}\right) A_{j}^{a}, \\
\delta_{f} A_{0}^{a} & =f^{j} \partial_{j} A_{0}^{a} .
\end{aligned}
$$

We find that

$$
\begin{gathered}
\delta_{f} L=\frac{\kappa}{4 \pi} \int_{D} d^{2} x \epsilon^{i j} \partial_{k}\left[f^{k}\left(\left(A_{i}+2 \alpha\left[Q, D_{i} Q\right]\right)^{a} \dot{A}_{j}^{a}+\alpha\left[Q, F_{i j}\right]^{a} \dot{Q}^{a}-A_{0}^{a} G_{i j}^{a}\right)\right] \\
=\frac{\kappa}{4 \pi} \oint_{\partial D} d \varphi f^{r}\left[\left(A_{r}+2 \alpha\left[Q, D_{r} Q\right]\right)^{a} \dot{A}_{\varphi}^{a}-\dot{A}_{r}^{a}\left(A_{\varphi}+2 \alpha\left[Q, D_{\varphi} Q\right]\right)^{a}\right. \\
\left.+\alpha \epsilon^{i j}\left[Q, F_{i j}\right]^{a} \dot{Q}^{a}-A_{0}^{a} \epsilon^{i j} G_{i j}^{a}\right] .
\end{gathered}
$$

In order to have Diff invariance, we must have $\delta_{f} L=\frac{d}{d t} X$, and there are two possible boundary conditions.

The first one is to choose $\left.f^{r}\right|_{\partial D}=0$ so that $X=0$. This boundary condition results in Diff only along the circle $(\partial D)$. The Noether charge for this Diff becomes

$$
C(f)=\frac{\partial L}{\partial \dot{A}_{i}^{a}} \delta_{f} A_{i}^{a}+\frac{\partial L}{\partial \dot{Q}^{a}} \delta_{f} Q^{a}=\gamma^{-1} \oint_{\partial D} d \varphi f^{\varphi}<J_{\varphi} J_{\varphi}>
$$


where in the second line we imposed the constraint $G_{i j}^{a}=0$ and its solution (5). Note that the above equation is the same as eq. (19), and we again get the Virasoro algebra without central charge. The second boundary condition corresponds to extending the solution (5) in the bulk to the boundary with an extra condition $A_{r}=\left.c\left[Q, \partial_{r} Q\right]\right|_{\partial D}=$ constant [20]. Then, the last line vanishes in (23), and it becomes $\frac{d X}{d t}$ with $X=-\gamma^{-1} \oint_{\partial D} d \varphi f^{r}<J_{r} J_{\varphi}>, \quad\left(J_{r}=\right.$ $\gamma\left[Q, \partial_{r} Q\right]=$ constant). After calculating the Noether charge, and imposing the solution, we get

$$
\begin{aligned}
C(f) & =\frac{\partial L}{\partial \dot{A}_{i}^{a}} \delta_{f} A_{i}^{a}+\frac{\partial L}{\partial \dot{Q}^{a}} \delta_{f} Q^{a}-X \\
& =\gamma^{-1} \oint_{\partial D} d \varphi\left(f^{\varphi}<J_{\varphi} J_{\varphi}>+2 f^{r}<J_{r} J_{\varphi}>\right)
\end{aligned}
$$

A straight forward computation yields

$$
\begin{aligned}
\{C(f), C(g)\}= & \gamma^{-1} \oint_{\partial D} d \varphi\left[\left[f^{\varphi}, g^{\varphi}\right]<J_{\varphi} J_{\varphi}>+2\left[f^{r \prime} g^{\varphi}-f^{\varphi} g^{r \prime}\right]<J_{r} J_{\varphi}>\right. \\
& \left.+2\left[f^{r \prime} g^{r}-f^{r} g^{r \prime}\right]\left(<J_{r} Q J_{r} Q>+\frac{1}{4}<J_{r} J_{r}>\right)\right]
\end{aligned}
$$

In general, this bracket does not satisfy the Jacobi identity for arbitrary $f^{r}$. One possible choice which satisfies the Jacobi identity is given by $\left.\left.f^{r}\right|_{\partial D} \propto \partial_{\varphi} f^{\varphi}\right|_{\partial D}$ and $\left.\left.g^{r}\right|_{\partial D} \propto \partial_{\varphi} g^{\varphi}\right|_{\partial D}$ (we choose the proportionality constant equal to 1) along with the condition that $J_{r}^{a}=$ $\gamma\left[Q, \partial_{r} Q\right]^{a}=$ constant. Then, the above equation (26) becomes the Virasoro algebra with classical central term;

$$
\{C(f), C(g)\}=C([f, g])-\frac{<J_{r} J_{r}>}{\gamma} \oint_{\partial D} d \varphi\left(f^{\prime \prime \prime} g-f g^{\prime \prime \prime}\right) .
$$

Thus, in contrast to the Diff along the circle $(\partial D)$, Diff which deforms across the boundary yields the Virasoro algebra with the classical third order derivative central term. This is similar to what happens in the pure Chern-Simons theory [6, 16].

The above results also suggest that an enlarged Virasoro algebra can be constructed. Let us consider the expression (25) directly on the boundary with some constant $J_{r}$. Then the $<J_{r} Q J_{r} Q>$ term in (26) does not become constant, and we must choose $\left.\left.f^{r}\right|_{\partial D} \propto f^{\varphi}\right|_{\partial D}$ and $\left.\left.g^{r}\right|_{\partial D} \propto g^{\varphi}\right|_{\partial D}$ in order to satisfy the Jacobi identity. This motivates introducing the elements of symmetric tensor product of $Q$ 's defined by

$$
\begin{aligned}
S(f) & =J_{r}^{\delta \alpha} J_{r}^{\gamma \beta} S_{\alpha \beta ; \gamma \delta}(f), \\
S_{\alpha \beta ; \gamma \delta}(f) & =2 \gamma^{-1} \oint_{\partial D} d \varphi f(\varphi)(Q \otimes Q)_{\alpha \beta ; \gamma \delta},
\end{aligned}
$$

and postulating the following enlarged Virasoro algebra;

$$
\begin{aligned}
& \{C(f), C(g)\}=C([f, g])+S([f, g])+k \oint_{\partial D} d \varphi\left(f^{\prime \prime \prime} g-f g^{\prime \prime \prime}\right), \\
& \{C(f), S(g)\}=-S\left((f g)^{\prime}\right), \quad\{S(f), S(g)\}=0,
\end{aligned}
$$

where $k=$ constant. The third order derivative central charge has been put in by hand and the above algebra satisfies the Jacobi identity. It is to be remarked that even if the Poisson 
bracket does not carry the central term classically, one can explicitly show that it arises from the standard quantum mechanical normal ordering [21] of $C$ and $S$. The chiral NLSM on the boundary has a central term with only first order derivative in (26). It would be interesting to find some explicit model in which the enlarged Virasoro algebra (29) can be realized classically.

\section{CONCLUSION}

We have constructed a boundary model in which the Chern-Simons gauge field and NLSM have bulk topological interaction with a manifest gauge invariance. We showed that the modified Gauss's law can be solved explicitly when the NLSM is defined on the Hermitian symmetric space, and the original bulk theory reduces to a boundary NLSM which has a time-independent diffeomorphism invariance. We find the coadjoint orbit method for NLSM particularly convenient in the process. We also calculated the symplectic structure and the diffeomorphism algebra. If we first calculate the Diff charge directly from the bulk action by the Noether procedure, and then reduce it to a boundary Diff charge using the solution of Gauss's law constraint, the ensuing Diff algebra which corresponds to deformation across the boundary yields the Virasoro algebra with classical central term. We also discovered an enlarged Virasoro algebra with elements of symmetric tensor product of $Q$ 's. It would be interesting to study the enlarged Virasoro algebra in detail, and to investigate whether the present result can find some application in the black hole physics.

\section{ACKNOWLEDGEMENTS}

I like to thank Joohan Lee for useful discussions, and Prof. R. Jackiw for his kind hospitality at CTP of MIT where part of this work was done. This work is supported by the Korea Research Foundation through program 1998-015-D00034. 


\section{REFERENCES}

[1] A. Achúcaro and P. K. Townsend, Phys. Lett. B 180 (1986) 89; E. Witten, Nucl. Phys. B 311 (1988) 46.

[2] M. Bañados, C. Teitelboim and J. Zanelli, Phys. Rev. Lett. 69 (1992) 1849.

[3] See S. Carlip, Class. Quant. Grav. 15 (1998) 3609; M. Bañados, hep-th/9901148; O. Aharony, S. S. Gubser, J. Maldacena, H. Ooguri, and Y. Oz, hep-th/9905111 and references therein.

[4] A. Strominger, J. High Energy Phys. 02 (1988) 009.

[5] J. D. Brown and M. Henneaux, Commun. Math. Phys. 104 (1986) 207.

[6] M. Bañados, Phys. Rev. D 52 (1996) 5816.

[7] S. Carlip, Phys. Rev. Lett. 82 (1999) 2828.

[8] R. Jackiw, and S. Templeton, Phys. Rev. D 23 (1981) 2291; J. F. Schonfeld, Nucl. Phys. B 18 (1981) 157; S. Deser, R. Jackiw, and S. Templeton, Ann. Phys. (N. Y.) 140 (1982) 372; (E) 185 (1988) 406.

[9] E. Witten, Commun. Math. Phys. 121 (1989) 351; G. Moore and N. Seiberg, Phys. Lett. B 220 (1989) 422; S. Elitzur, G. Moore, A. Schwimmer, and N. Seiberg, Nucl. Phys. B 326 (1989) 108; M. Bos and V. P. Nair, Int. J. Mod. Phys. A 5 (1990) 959.

[10] A. M. Perelomov, Phys. Rep. 146 (1987) 135.

[11] P. Oh and Q-H. Park, Phys. Lett. B 383 (1996); Phys. Lett. B 400 (1997) 157; (E) 416 (1998) 452; P. Oh, J. Phys. A: Math. Gen. 31 (1998) L325; Rept. Math. Phys. 43 (1999) 271.

[12] L. D. Faddeev and R. Jackiw, Phys. Rev. Lett. 60 (1988) 1692; M. Henneaux and C. Teitelboim, Quantization of Gauge Systems (Princeton Univ. Press, 1992)

[13] A. P. Fordy and P. P. Kulish, Commum. Math. Phys. 89 (1983) 427.

[14] A. Jevicki and N. Papanicolaou, Phys. Lett. B 78 (1978) 438; A. Bassetto and G. Nardelli, Phys. Rev. D 57 (1998) 2628.

[15] Note that when $\alpha \rightarrow \infty, c=-1$, and $D_{\mu} Q=0$. Then, the Gauss's law constraint (四) can have extra solution $A_{i}=d\left[Q, \partial_{i} Q\right]+a_{i} Q$, where $a_{i}$ is an arbitrary vector field ( $\mathrm{Y}$. Nambu, Int. J. Thoer. Phys. 17 (1978) 287 ). However, such solution does not admit a complete reduction to boundary theory, and will not be considered here.

[16] P. Oh and M.-I. Park, Mod. Phys. Lett. A 14 (1999) 231; M.-I. Park, Nucl. Phys. B 544 (1999) 377.

[17] D. Bak, R. Jackiw and S.-Y. Pi, Phys. Rev. D 49 (1994) 6778.

[18] J.-M. Maillet, Phys. Lett. B 162 (1985) 137; M. J. Bowick, D. Karabali, and L. C. R. Wijewardhana, Nucl. Phys. B 271 (1986) 417.

[19] M. Forger, J. Laartz and U. Schäper, Commun. Math. Phys. 146 (1992) 397.

[20] This condition forces the variables $Q^{a}$ 's to a subset of all possible configurations on the boundary. A more detailed study will be addressed elsewhere.

[21] P. Goddard and D. Olive, Int. J. Mod. Phys. A 1 (1986) 303. 